\title{
EFECTIVIDAD EN ATAQUE Y CONTRAATAQUE. COMPARACIÓN ENTRE EL CAMPEONATO MUNDIAL DE VOLEIBOL FEMENINO 2002 Y JUEGOS NACIONALES 2004
}

\author{
Fernando Ubiratan da Silveira y Walter Salazar Rojas \\ Escuela de Educación Física y Deportes \\ Maestría en Ciencias del Movimiento Humano \\ Universidad de Costa Rica \\ e-mail:voleibr@hotmail.com
}

\begin{abstract}
Resumen
Da Silveira, F. y Salazar Rojas, W. (2007). Efectividad en ataque y contraataque. Comparación entre el Campeonato Mundial de Voleibol 2002 y Juegos Deportivos Nacionales de Costa Rica 2004. Revista de Ciencias del Ejercicio y la Salud 5(1), 19-23. Esta investigación presenta y compara la efectividad entre ataque y contraataque, las posiciones de ataque y velocidades de jugadas más efectivas para cada complejo en dos competiciones de distintos niveles. Los equipos observados fueron: 1. Selecciones principales de Rusia, Cuba y Brasil (3er, $5^{\circ}$ y $7^{\circ}$ puestos respectivamente), en el Campeonato Mundial de Voleibol 2002. 2. Selecciones de los Cantones de Santa Bárbara, San José y Alajuela (1er, $2^{\circ}$ y $3^{\circ}$ puestos respectivamente), en los Juegos Nacionales de Costa Rica 2004. Los resultados demuestran que la efectividad en los equipos del Campeonato Mundial (CM) es de $80,6 \%$ y $33,54 \%$ para ataque y contraataque respectivamente, mientras que en los equipos de Juegos Nacionales (JN) la efectividad está en $41,18 \%$ y $39,28 \%$. Las jugadas de velocidad media fueron las más efectivas tanto para el ataque cuanto para el contraataque en el CM, mientras que para $\mathrm{JN}$ fueron las jugadas de velocidad rápida. Las posiciones 1 y 2 fueron las más efectivas para CM tanto para ataque cuanto para contraataque, mientras que para $\mathrm{JN}$ fueran las posiciones 4 para ataque y 3 para el contraataque.

PALABRAS CLAVES: voleibol, efectividad, ataque-contraataque, niveles.
\end{abstract}

\section{INTRODUCCIÓN}

La preparación de un equipo de voleibol para un partido consiste en la utilización por el entrenado, de todos los medios posibles para sus atletas (su equipo) autónomos, capaces de reaccionar de una manera precisa y eficaz a las situaciones creadas por el adversario (Hernández, 2000).

Según Cordeiro y Nascimento (1996), diversos caminos pueden ser seguidos por el entrenador para la preparación de un equipo. Entretanto, una preparación detallada necesitará de una secuencia lógica de acciones para la toma de decisiones que consiste en: recolección de datos; análisis de estos datos y toma de decisiones; elaboración del plan de juego y entrenamiento final (Loret, 1999).
Desde hace algunos años se viene utilizando una terminología para determinar los dos momentos del juego de voleibol: 1 . Ataque, que consiste en Recepción-PaseRemate y 2. Contraataque, que consiste en Bloqueo-Defensa de Campo-Pase-Remate. Esta división se basa en la necesidad de entrenar, analizar y evaluar este deporte teniendo en cuenta quién posee el servicio (Hernández, 2000).

Debido al creciente desarrollo fisco, técnico y táctico de los equipos, el ataque se impone con gran ventaja sobre la defensa, por eso, desde hace más de 25 años, se han adoptado diferentes reglas y modificaciones para tratar de hacer menor ese desequilibrio, algunas de estas modificaciones fueron: invasión de manos y brazos en la acción del bloqueo hacia el terreno contrario, permitir golpear el balón con cualquiera parte del cuerpo, introducción del libero, entre otras. 
No obstante a las modificaciones de las reglas del juego, ésta problemática continúa vigente manteniendo un gran predominio del las acciones de ataque sobre las acciones de defensa.

Según Hernández (2000), la efectividad en ataque oscila entre un 75 y $85 \%$, en estudio semejante Cordeiro y Nacimento (1997), reportaran una efectividad de $70 \%$, lo que demostró la gran importancia de que un equipo posea buena efectividad en ataque.

El contraataque presenta gran complejidad ya que en la mayoría de los casos el balón regresa en forma de violento remate ejecutado por el adversario, o sea, de manera difícil de defenderse. En muchas acciones es necesario pasar de la defensa al ataque para obtener el punto, por eso su gran complejidad. Sus formas esenciales se notan claramente en dos situaciones: 1. Bloqueo; 2. Defensa de campo y Transición para el remate. Según Hernández (2000), en alto nivel la mayoría de los puntos en contraataque provienen de la segunda variante, o sea, defensa y transición para el contraataque.

Entretanto, en la literatura revisada los datos presentados referentes a efectividad en ataque y contraataque no parecen ser fruto de investigación científica, sino de experiencias personales, además, no se ha podido encontrar datos referentes a la efectividad en competiciones de niveles inferiores.

Por eso, la presente investigación pretende hacer una comparación entre los distintos niveles, referentes a la efectividad entre ataque y contraataque, además, averiguar las posiciones y velocidades de jugadas más efectivas para cada complejo.

\section{METODOLOGÍA}

Sujetos: los sujetos observados fueron: 1.Selecciones principales femeninas de Rusia, Cuba y Brasil (3er, $5^{\circ}$ y $7^{\circ}$ puestos respectivamente), en el Campeonato Mundial de Voleibol 2002 (CM). 2.Selecciones femeninas juveniles de los Cantones de Santa Bárbara, San José y Alajuela (1er, $2^{\circ}$ y $3^{\circ}$ puestos respectivamente), en los Juegos Deportivos Nacionales de Costa Rica del $2004(\mathrm{JN})$.

Instrumento de medición: en ambas competiciones los equipos fueran filmados por los investigadores con cámara VHS; posteriormente los datos fueron transferidos para hojas de cotejo (tablas) donde se registraban las acciones.

Procedimientos: fueron confeccionadas tablas donde se les apuntaban:

a) las jugadas efectivas (que generaban punto directo),

b) las jugadas no efectivas (que permitían el proseguimiento del juego);

En dos situaciones:

a) Ataque (primera acción de remate que se realiza después del saque)

b) Contraataque (acciones que siguen después del ataque)

En todas las posiciones de la cancha $(1,2,3,4,5$ y 6$)$;

Además, se registraba también la velocidad de la jugada:

(R) Rápida

(M) Media

(L) Lenta.

- Cada equipo tuvo evaluado el siguiente número de sets:

Grupo Campeonato Mundial:

- Brasil: 3 sets

- Cuba: 5 sets

- Rusia: 4 sets

Grupo Juegos Nacionales

- Alajuela: 3 sets

- San José: 4 sets

- Santa Bárbara: 3 sets. 
Análisis estadístico: para el análisis de los datos fue utilizada solamente estadística descriptiva, donde se pudo calcular los porcentajes de efectividad para comparación entre los complejos, las zonas preferidas de remate, las velocidades de jugadas y los grupos.

\section{RESULTADOS}

Para el cálculo del porcentaje de efectividad se sacó la diferencia entre los remates ejecutados y los que produjeran puntos en todos los partidos de cada uno de los grupos. Además, para hacer una comparación paralela, se hizo el siguiente cálculo: total de acciones dividido por el número de sets jugados en cada grupo (12 en $\mathrm{CM}$ y 10 en JN).

El gráfico 1 muestra la efectividad entre ataque y contraataque, donde se puede verificar que en el ataque el grupo CM presenta una efectividad mayor que en el grupo JN. Entretanto, esa tendencia no se presentó en la efectividad del contraataque, donde en el grupo $\mathrm{JN}$ fue ligeramente más efectivo. Además, en el gráfico 2 se puede verificar que las cantidades de acciones por set son significativamente mayores en el grupo $\mathrm{CM}$ para el ataque y que para el contraataque esa relación está invertida, o sea, el grupo $\mathrm{JN}$ presenta una cantidad de acciones ligeramente mayor.

Gráfico 1. Efectividad del ataque y contraataque en porcentaje de los grupos Campeonato Mundial y Juegos Nacionales

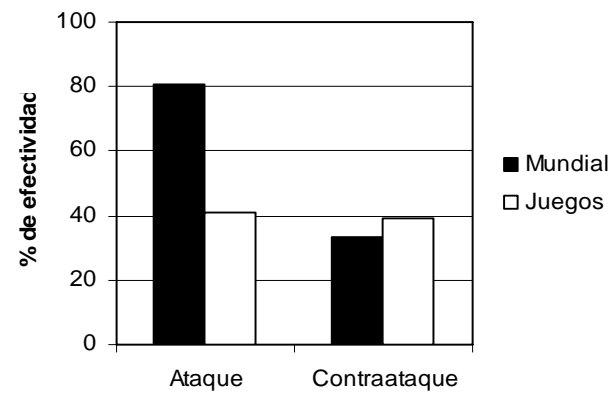

Gráfico 2. Acciones por sets del ataque y contraataque de los grupos Campeonato Mundial y Juegos Nacionales

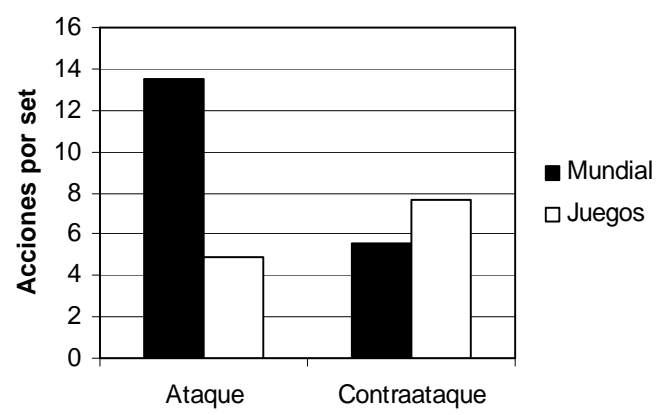

En la comparación de la efectividad con respecto a la velocidad de la jugada en el ataque, se puede observar en el gráfico 3 , que las jugadas de velocidad media y rápida fueran más efectivas para los 2 grupos y que fueron aún más efectivas en el grupo CM. Cuando, observamos las cantidades de acciones realizadas por set podemos verificar que en el grupo JN las principales acciones son provenientes de jugadas lentas (gráfico 4).

Gráfico 3. Efectividad del ataque en porcentaje
según la velocidad de jugada de los grupos
Campeonato Mundial y Juegos Nacionales

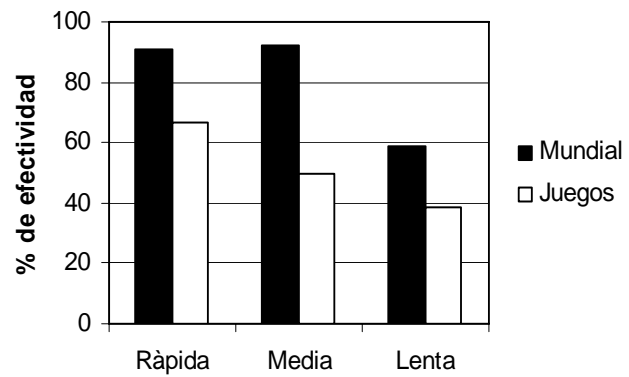

Gráfico 4. Acciones por sets del ataque según la velocidad de jugada de los grupos Campeonato Mundial y Juegos Nacionales

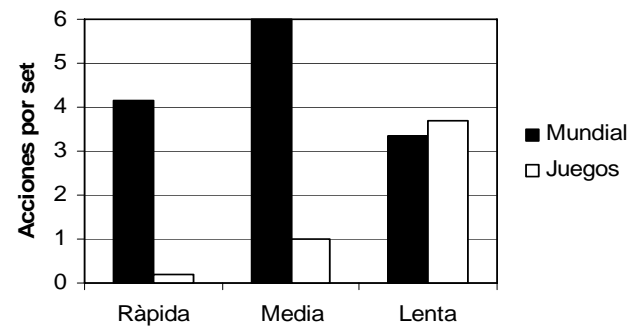


Entretanto, en el contraataque (gráfico 5) el grupo $\mathrm{JN}$ presento una efectividad más alta para todas las velocidades de jugadas probablemente debido a la mayor cantidad de acciones realizadas en ese complejo (gráfico 6).

Gráfico 5. Efectividad del contraataque en porcentaje según la velocidad de jugada de los grupos Campeonato Mundial y Juegos Nacionales

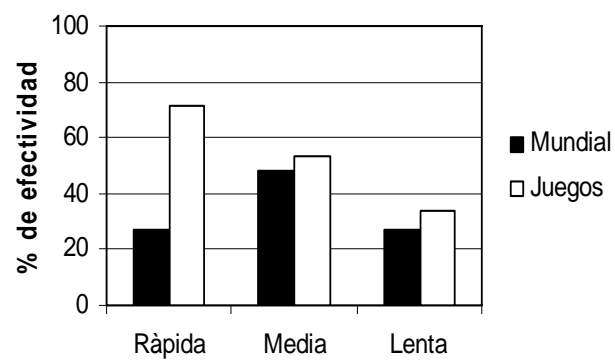

Gráfico 6. Acciones por set del contraataque según la velocidad de jugada de los grupos Campeonato Mundial y Juegos Nacionales

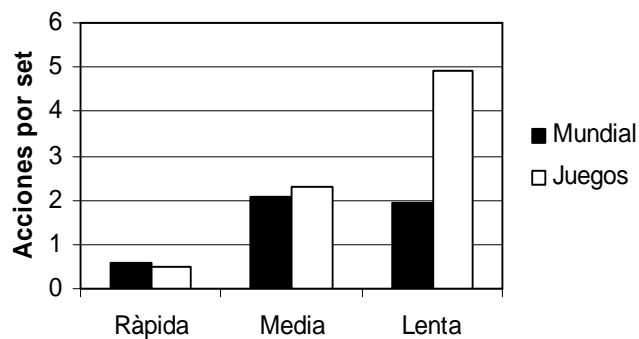

En los gráficos 7 y 9 se puede observar la efectividad de los grupos por posición del remate, donde en los dos grupos hubo mayor cantidad de acciones por set en posiciones delanteras $(2 ; 3 \mathrm{y} 4)$ en ataque y contraataque (gráficos 8 y 10). En el grupo CM la efectividad se ve más alta y las acciones mejor distribuidas, mientras que en el grupo $\mathrm{JN}$ concentraban prácticamente todas las acciones en ataque y contraataque por las posiciones delanteras.
Gráfico 7. Efectividad del ataque en porcentaje según la posición del remate en los grupos Campeonato Mundial y Juegos Nacionales

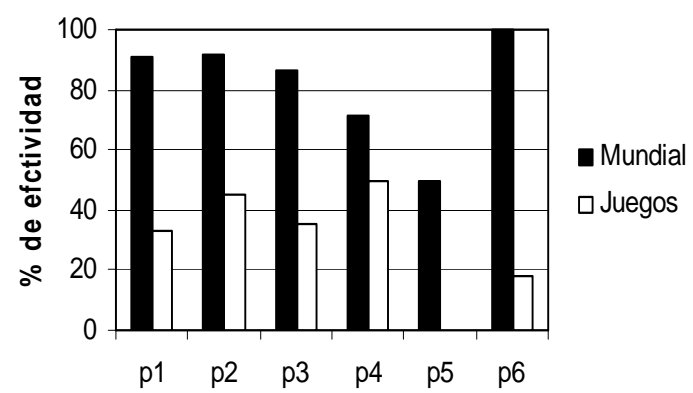

Gráfico 8. Acciones por set del ataque según la posición del remate en los grupos Campeonato Mundial y Juegos Nacionales

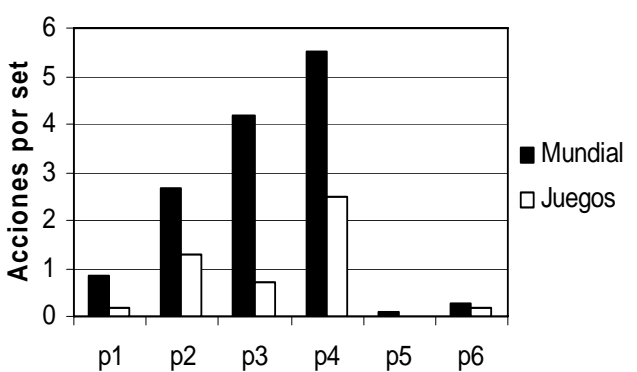

Gráfico 9. Efectividad del contraataque en porcentaje según la posición del remate en los grupos Campeonato Mundial y Juegos Nacionales

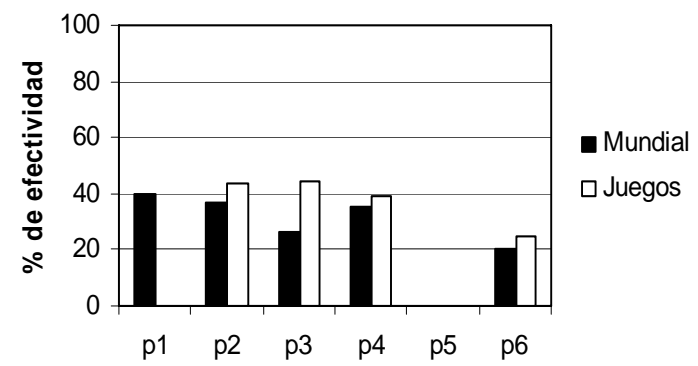


Gráfico 10. Acciones por set del contraataque según la posición del remate en los grupos Campeonato Mundial y Juegos Nacionales

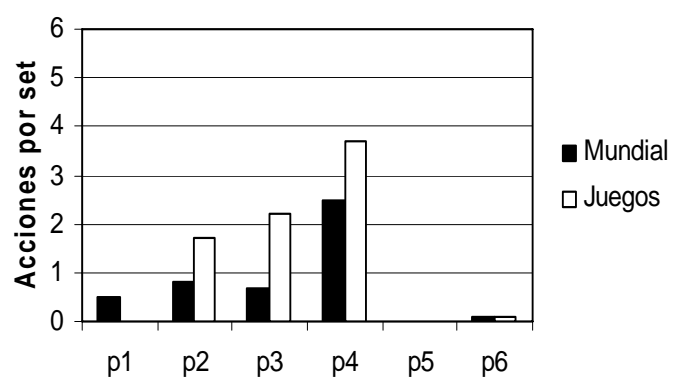

\section{DISCUSIÓN}

Para el análisis de la efectividad en los grupos hay que tener cierto cuidado, pues en algunas situaciones el promedio de la cantidad de acciones por set (Gráficos $2 ; 4 ; 6$; 8 ; y 10) son pequeñas y en algunos casos inexistentes, lo que puede generar interpretaciones equivocadas.

El resultado encontrado para la efectividad en el ataque (80\%) está de acuerdo con los datos reportados por Hernández (2000), (75-85\%) para equipos de alto nivel. Esa mayor efectividad en el ataque para el grupo CM se debe probablemente debido a mejor calidad técnica y física de los sujetos, o sea, cuando los sujetos son capaces de realizar una buena recepción del saque adversario, posibilita condiciones óptimas para que su colocador pueda acomodar el balón de forma ideal y que el rematador logre marcar el punto.

La mayor efectividad en contraataque encontrada para el grupo JN es probablemente debido a la baja efectividad en ataque, lo que posibilitó una mayor cantidad de acciones en contraataque y por consecuencia más puntos marcados en ese complejo.

Según la velocidad de jugada, en el ataque las jugadas lentas son las menos efectivas para los dos grupos, o esa, cuanto más lenta es la jugada, mayor es la posibilidad del equipo adversario defenderse debido al mayor tiempo disponible para que los defensores se ubiquen correctamente en sus posiciones y logren éxito.

En el contraataque se pudo observar una mayor tendencia de los dos grupos (Tabla $5)$ por rematar en las posiciones delanteras (2; 3 y 4), donde se puede decir que la diferencia de nivel técnico y físico no fue factor importante en la variabilidad de jugadas en contraataque. Pero, en el ataque el grupo JN prácticamente solo utiliza las posiciones delanteras en ataque (Gráfico 4) posiblemente por ser las posiciones más fáciles de rematar, esto hace que el juego sea más previsible.

Finalmente, podemos concluir con los resultados del presente estudio, que en la comparación entre los dos grupos, CM y JN, las diferencias en los porcentajes de efectividad de los remates se dieran debido a las diferencias técnicas y físicas de las jugadoras. Donde para el grupo CM que presentó mayor efectividad en el ataque demostrando que cuanto mejor la calidad técnica y física de las jugadoras, mayor será la variación y velocidad de las jugadas, aumentando la efectividad y la prevalecía del ataque sobre la defensa.

\section{REFERENCIAS}

Cordeiro, C. y Nascimento, L. (1996). Coleta e análise de dados são essenciais na preparação da equipe. Volei Técnico, Confederação Brasileira de Volley-ball. 7, 9-15.

Hernández, A. (2000). Manual de entrenadores FIVB. Curso SAP.

Loret, M. (1999). Los coeficientes ofensivos y defensivos. Una aportación al estudio práxico de los deportes de equipo. Apunts, Educación Física y Deportes 55, 68-76. 